\title{
Stabilizers for Nondegenerate Matrices of Boundary Format and Steiner Bundles
}

\author{
Carla DIONISI \\ Dipartimento di Matematica Applicata "G. Sansone" \\ Via S. Marta, 3 \\ I-50139, Firenze, Italy \\ dionisi@dma.unifi.it
}

Recibido: 25 de Febrero de 2002

Aceptado: 26 de Abril de 2004

\begin{abstract}
In this paper nondegenerate multidimensional matrices of boundary format in $V_{0} \otimes \cdots \otimes V_{p}$ are investigated by their link with Steiner vector bundles on product of projective spaces. For any nondegenerate matrix $A$ the stabilizer for the $\mathrm{SL}\left(V_{0}\right) \times \cdots \times \mathrm{SL}\left(V_{p}\right)$-action, $\operatorname{Stab}(A)$, is completely described. In particular we prove that there exists an explicit action of SL(2) on $V_{0} \otimes \cdots \otimes V_{p}$ such that $\operatorname{Stab}(A)^{0} \subseteq \operatorname{SL}(2)$ and the equality holds if and only if $A$ belongs to a unique $\mathrm{SL}\left(V_{0}\right) \times \cdots \times \mathrm{SL}\left(V_{p}\right)$-orbit containing the identity matrices, according to [1].
\end{abstract}

Key words: Vector bundles, multidimensional matrices, theory of invariants

2000 Mathematics Subject Classification: 14F05; 15A72

\section{Introduction}

Let $V_{j}$ be a complex vector space of dimension $k_{j}+1$ for $j=0, \ldots, p$ with $k_{0}=$ $\max _{i}\left\{k_{i}\right\}$. Gelfand, Kapranov and Zelevinsky in [5] proved that the dual variety of the Segre product $\mathbb{P}\left(V_{0}\right) \times \cdots \times \mathbb{P}\left(V_{p}\right)$ is a hypersurface in $\left(\mathbb{P}^{\left(k_{0}+1\right) \cdots\left(k_{p}+1\right)-1}\right)^{\vee}$ if and only if $k_{0} \leq \sum_{i=1}^{p} k_{i}$. The defining equation of this hypersurface is called the hyperdeterminant of format $\left(k_{0}+1\right) \times \cdots \times\left(k_{p}+1\right)$ and is denoted by Det. Moreover the hyperdeterminant is a homogeneous polynomial function on $V_{0}^{\vee} \otimes \cdots \otimes V_{p}^{\vee}$ so that the condition Det $A \neq 0$ is meaningful for a $(p+1)$-dimensional matrix $A \in \mathbb{P}\left(V_{0} \otimes \cdots \otimes V_{p}\right)$ of format $\left(k_{0}+1\right) \times \cdots \times\left(k_{p}+1\right)$. The hyperdeterminant is an invariant for the natural action of $\mathrm{SL}\left(V_{0}\right) \times \cdots \times \mathrm{SL}\left(V_{p}\right)$ on $\mathbb{P}\left(V_{0} \otimes \cdots \otimes V_{p}\right)$, and, in particular, if Det $A \neq 0$ then $A$ is semistable for this action. 
We denote by $\operatorname{Stab}(A) \subset \mathrm{SL}\left(V_{0}\right) \times \cdots \times \mathrm{SL}\left(V_{p}\right)$ the stabilizer subgroup of $A$ and by $\operatorname{Stab}(A)^{0}$ its connected component containing the identity. The stabilizer are well known for $p \leq 1$ (in this case there is always a dense orbit and the orbits are determined by the rank), so that in this paper we assume $p \geq 2$.

It easy to check (see [12], [3]) that the degenerate matrices fill an irreducible variety of codimension $k_{0}-\sum_{i=1}^{p} k_{i}+1$ and if $k_{0}<\sum_{i=1}^{p} k_{i}$ then all matrices are degenerate. We will assume from now on that $A$ is of boundary format i.e., that $k_{0}=\sum_{i=1}^{p} k_{i}$. (A self-contained approach to hyperdeterminant of boundary format matrices can be found in [3].)

For multidimensional boundary format matrices the classical definitions of triangulable, diagonalizable and identity matrices can be easily reformulate in the natural way as follows

Definition 1.1. A $(p+1)$-dimensional matrix of boundary format $A \in V_{0} \otimes \cdots \otimes V_{p}$ is called

(i) triangulable if $\forall j$ there exists a basis $e_{0}^{(j)}, \ldots, e_{k_{j}}^{(j)}$ of $V_{j}$ such that

$$
A=\sum a_{i_{0}, \ldots, i_{p}} e_{i_{0}}^{(0)} \otimes \cdots \otimes e_{i_{p}}^{(p)} \quad \text { where } a_{i_{o}, \ldots, i_{p}}=0 \text { for } i_{0}>\sum_{t=1}^{p} i_{t}
$$

(ii) diagonalizable if there exists a basis $e_{0}^{(j)}, \ldots, e_{k_{j}}^{(j)}$ of $V_{j}$ such that

$$
A=\sum a_{i_{0}, \ldots, i_{p}} e_{i_{0}}^{(0)} \otimes \cdots \otimes e_{i_{p}}^{(p)} \quad \text { where } a_{i_{o}, \ldots, i_{p}}=0 \text { for } i_{0} \neq \sum_{t=1}^{p} i_{t}
$$

(iii) an identity if there exists a basis $e_{0}^{(j)}, \ldots, e_{k_{j}}^{(j)}$ of $V_{j}$ such that

$$
A=\sum a_{i_{0}, \ldots, i_{p}} e_{i_{0}}^{(0)} \otimes \cdots \otimes e_{i_{p}}^{(p)}
$$

where

$$
a_{i_{o}, \ldots, i_{p}}=\left\{\begin{array}{lll}
0 & \text { for } & i_{0} \neq \sum_{t=1}^{p} i_{t} \\
1 & \text { for } & i_{0}=\sum_{t=1}^{p} i_{t} .
\end{array}\right.
$$

Ancona and Ottaviani in [1], considering the natural action of $\mathrm{SL}\left(V_{0}\right) \times \cdots \times \mathrm{SL}\left(V_{p}\right)$ on $\mathbb{P}\left(V_{0} \otimes \cdots \otimes V_{p}\right)$, analyze these properties from the point of view of Mumford's Geometric Invariant Theory.

In the same aim, the main result of this paper is the following:

Theorem 1.2. Let $A \in \mathbb{P}\left(V_{0} \otimes \cdots \otimes V_{p}\right)$ be a boundary format matrix with Det $A \neq$ 0 .Then there exists a 2-dimensional vector space $U$ such that $\operatorname{SL}(U)$ acts over $V_{i} \simeq$ 
$S^{k_{i}} U$ and according to this action on $V_{0} \otimes \cdots \otimes V_{p}$ we have $\operatorname{Stab}(A)^{0} \subseteq \operatorname{SL}(U)$. Moreover the following cases are possible

$$
\operatorname{Stab}(A)^{0} \simeq\left\{\begin{array}{l}
0 \\
\mathbb{C} \\
\mathbb{C}^{*} \\
\operatorname{SL}(2) \quad \text { (this case occurs if and only if } A \text { is an identity) }
\end{array}\right.
$$

Remark 1.3. We emphasize that $\mathrm{SL}\left(V_{0}\right) \times \cdots \times \mathrm{SL}\left(V_{p}\right)$ is a "big" group, so it is quite surprising that the stabilizer found lies always in the 3-dimensional group $\operatorname{SL}(U)$ without any dependence on $p$ and on $\operatorname{dim} V_{i}$.

The maximal stabilizer is obtained by the "most symmetric" class of matrices corresponding to the identity matrices. Under the identifications $V_{i}=S^{k_{i}} U$ the identity is given by the natural map

$$
S^{k_{1}} U \otimes \cdots \otimes S^{k_{p}} U \rightarrow S^{k_{0}} U
$$

which is defined under the assumption $k_{0}=\sum k_{i}$. This explains again why the condition of boundary format is so important.

Ancona and Ottaviani in [1] prove Theorem 1.2 for $p=2$. We generalize their proof by using the correspondence between nondegenerate boundary format matrices and vector bundles on a product of projective spaces.

Indeed, for any fixed $j \neq 0$, a $(p+1)$-dimensional matrix $A \in V_{0} \otimes \cdots \otimes V_{p}$ of format $\left(k_{0}+1\right) \times \cdots \times\left(k_{p}+1\right)$ defines a sheaf morphism $f_{A}^{(j)}$ on the product $X=\mathbb{P}^{k_{1}} \times \cdots \times \widehat{\mathbb{P}^{k_{j}}} \times \cdots \times \mathbb{P}^{k_{p}}$

$$
\mathcal{O}_{X} \otimes V_{0}^{\vee} \stackrel{f_{A}^{(j)}}{\longrightarrow} \mathcal{O}_{X}(1, \ldots, 1) \otimes V_{j}
$$

and it is easy to prove the following

Proposition 1.4 ([1], [2]). If a matrix $A$ is of boundary format, then $\operatorname{Det} A \neq 0$ if and only if for all $j \neq 0$ the morphism $f_{A}^{(j)}$ is surjective $\left(\right.$ so $S_{A}^{\vee(j)}=\operatorname{Ker} f_{A}^{(j)}$ is a vector bundle of rank $k_{0}-k_{j}$ ).

In the particular case $p=2$ the (dual) vector bundle $S_{A}{ }^{(1)}$ (or $S_{A}{ }^{(2)}$ ) lives on the projective space $\mathbb{P}^{n}, n=k_{2}$ (or $n=k_{1}$ ) and it is a Steiner bundle as defined in [4] (this case has been investigate in [1]). We shall refer to $S_{A}^{(j)}$ with the name Steiner also for $p \geq 3$.

The main new technique introduced in this paper is the use of jumping hyperplanes for bundles on the product of $(p-1)$ projective spaces. For $p \geq 2$ there are two natural ways to introduce them; by the above correspondence, they translate into two different conditions on the associated matrix and that we call weak and strong (see definition 2.1 and 2.6). They coincide when $p=2$. 
Moreover, the loci of weak and strong jumping hyperplanes are invariant for the action of $\mathrm{SL}\left(V_{0}\right) \times \cdots \times \mathrm{SL}\left(V_{p}\right)$ on matrices. By investigating these invariants we derive the proof of Theorem 1.2 and also we obtain a characterization of a particular class of bundles called Schwarzenberger bundles (see [10] for the original definition in the case $p=2$ ). Schwarzenberger bundles correspond exactly to such matrices $A$ which verify the equality $\operatorname{Stab}(A)^{0}=\mathrm{SL}(2)$ in Theorem 1.2, called identity matrices.

I would like to thank G. Ottaviani for his invaluable guidance and the referee for useful suggestions to improve this note.

\section{Jumping hyperplanes and stabilizers}

Let $p=2$ and $S:=S^{1}$ be the Steiner bundle on $\mathbb{P}\left(V_{2}\right)$ defined by a matrix $A \in$ $V_{0} \otimes V_{1} \otimes V_{2}$ of boundary format, an hyperplane $h \in \mathbb{P}\left(V_{2}^{\vee}\right)$ is an unstable hyperplane of $S$ if $h^{0}\left(S_{\mid h}^{\vee}\right) \neq 0$ (see [1]). By abuse of notations we identify an hyperplane $h \in \mathbb{P}\left(V_{2}^{\vee}\right)$ with any vector $h^{\prime} \in V_{2}$ such that $\left\langle h^{\prime}\right\rangle=h$.

In particular, $\mathcal{H}^{0}\left(S^{\vee}(t)\right)$ identifies to the space of $\left(k_{0}+1\right) \times 1$-column vectors $v$ with entries in $S^{t} V_{2}$ such that $A v=0$, and a hyperplane $h$ is unstable for $S$ if and only if there are nonzero vectors $v_{0}$ of size $\left(k_{0}+1\right) \times 1$ and $v_{1}$ of size $\left(k_{1}+1\right) \times 1$ both with constant coefficients such that

$$
A v_{0}=v_{1} h
$$

the tensor $\mathcal{H}=v_{0} \otimes v_{1}$ is called an unstable (or jumping) hyperplane for the matrix $A$.

For $p \geq 3$ there are at least two ways to define a jumping hyperplane. We will call them weak and strong jumping hyperplanes.

Definition 2.1. $\mathcal{H}=v_{0} \otimes v_{j} \otimes h \in V_{0} \otimes V_{j} \otimes \widehat{V}^{j}$ (where $\left.\widehat{V}^{j}=V_{1} \otimes \cdots \otimes \widehat{V}_{j} \otimes \cdots \otimes V_{p}\right)$ is a $(j)$-weak jumping hyperplane for $A$ if $\exists v_{0}, w_{1}, \ldots, w_{k_{0}}$ basis of $V_{0}$ such that

$$
A=v_{0} \otimes v_{j} \otimes h+\sum_{i=1}^{k_{0}} w_{i} \otimes \cdots
$$

where $h \in \widehat{V}^{j}$ generate an hyperplane for $\mathbb{P}^{k_{1}} \times \cdots \times \widehat{\mathbb{P}^{k_{j}}} \times \cdots \times \mathbb{P}^{k_{p}} \subset \mathbb{P}\left(V_{1} \otimes \cdots \otimes\right.$ $\left.\widehat{V_{j}} \otimes \cdots \otimes V_{p}\right)$ (that, by abuse of notations, we call also $h$ ).

Remark 2.2. The expression (3) means, as in the case $p=2$, that $H^{0}\left(\operatorname{Ker} f_{A_{\mid h}}^{(j)}\right) \neq 0$ (i.e., by definition, $h$ is a jumping hyperplane for the bundle $S_{A}{ }^{(j)}$ ).

If $\mathcal{H}=v_{0} \otimes v_{j}$ is a $(j)$-weak jumping hyperplane for $A$ then the map:

$$
\begin{aligned}
V_{0} \otimes \cdots \otimes V_{p} & \rightarrow\left(V_{0} /\left\langle v_{0}\right\rangle\right) \otimes \cdots \otimes\left(V_{j} /\left\langle v_{j}\right\rangle\right) \otimes \cdots \otimes V_{p} \\
A & \mapsto A^{\prime}{ }_{j}
\end{aligned}
$$

gives an elementary transformation [8]. 
Remark 2.3. $A^{\prime}{ }_{j}$ is again of boundary format. In particular, after a basis has been chosen, $A^{\prime}{ }_{j}$ is obtained by deleting two directions in $A$.

Proposition 2.4. If $A^{\prime}{ }_{j}$ is defined as above

$$
\operatorname{Det} A \neq 0 \Rightarrow \operatorname{Det} A^{\prime}{ }_{j} \neq 0
$$

Proof. If $X:=\mathbb{P}^{k_{1}} \times \cdots \times \widehat{\mathbb{P}^{k_{j}}} \times \cdots \times \mathbb{P}^{k_{p}}$ and $h$ is the hyperplane defined in 2.1 associated to $\mathcal{H}$, the map $S_{A}^{(j)} \rightarrow \mathcal{O}_{h}$ induced by a non zero section of $S_{A}{ }^{(j)}$ is surjective (the same proof of [14, prop. 2.1] works).

Since $\operatorname{codim} h=1$, then its kernel $S^{\prime(j)}$ is locally free sheaf [11] of rank $k_{0}-k_{j}-1$ on $X$ and it is the Steiner bundle associated to the matrix $A^{(j)}$ as the snake-lemma applied to the following exact diagram shows

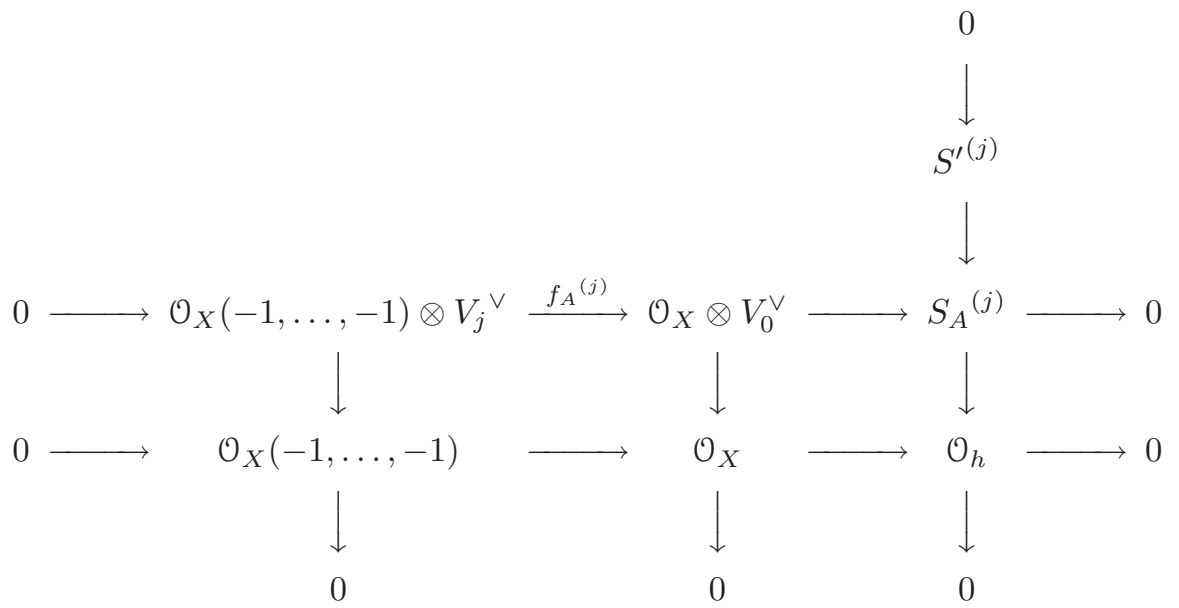

i.e., $S^{\prime(j)}=S_{A_{j}^{\prime}}^{(j)}$ and by Proposition 1.4 the result follows.

Remark 2.5. If $W\left(S_{A}^{(j)}\right)$ is the set of jumping hyperplanes of the bundle $S_{A}^{(j)}$, then the exact sequence (dual to the last column of the above diagram)

$$
0 \rightarrow S_{A}^{(j)^{\vee}} \rightarrow S_{A_{j}^{\prime}}^{(j)^{\vee}} \rightarrow \mathcal{O}_{X}(1, \ldots, 1) \rightarrow 0
$$

shows that $W\left(S_{A}^{(j)}\right) \subset W\left(S_{A_{j}^{\prime}}^{(j)}\right) \cup\{h\}$

Definition 2.6. $\mathcal{H}=v_{0} \otimes v_{1} \otimes \cdots \otimes v_{p}$ is a strong jumping hyperplane for $A$ if $\exists v_{0}, w_{1}, \ldots, w_{k_{0}}$ basis of $V_{0}$ such that

$$
A=v_{0} \otimes v_{1} \otimes \cdots \otimes v_{p}+\sum_{i=1}^{k_{0}} w_{i} \otimes \cdots
$$


Remark 2.7. If $\mathcal{H}$ is a strong jumping hyperplane then $\mathcal{H}$ defines a $(j)$-weak jumping hyperplane for all $j=1, \ldots, p$; in particular for a strong jumping hyperplane there are many elementary transformations.

Remark 2.8. For $p=2$ the notations of strong jumping hyperplane and of weak jumping hyperplane coincide with each other (see [1]).

Example 2.9 (the identity). Fixed a basis $e_{0}^{(j)}, \ldots, e_{k_{j}}^{(j)}$ in $V_{j}$ for all $j$, the identity matrix is represented by

$$
I:=\sum_{\substack{i_{0}=i_{1}+\cdots+i_{p} \\ 0 \leq i_{j} \leq k_{j}}} e_{i_{0}}^{(0)} \otimes \cdots \otimes e_{i_{p}}^{(p)} .
$$

Let $t_{0}, \ldots, t_{k_{0}}$ be any distinct complex numbers. Let $w$ be the $\left(k_{0}+1\right) \times\left(k_{0}+1\right)$ Vandermonde matrix whose $(i, j)$ entry is $t_{j}^{(i-1)}$, so acting with $w$ over $V_{0}$, we have:

$$
e_{j}^{(0)}=\sum_{s=0}^{k_{0}} \bar{e}_{s}^{(0)} t_{s}^{j}
$$

Then substituting

$$
\begin{aligned}
I & =\sum_{\substack{i_{0}=i_{1}+\cdots+i_{p} \\
s=0, \ldots k_{0}}} \bar{e}_{i_{0}}^{(0)} t_{s}^{i_{0}} \otimes e_{i_{1}}^{(1)} \otimes \cdots \otimes e_{i_{p}}^{(p)} \\
& =\sum_{s=0}^{k_{0}} \bar{e}_{s}^{0} \otimes\left(\sum_{i_{1}=0}^{k_{1}} e_{i_{1}}^{(1)} t_{s}^{i_{1}}\right) \otimes \cdots \otimes\left(\sum_{i_{p}=0}^{k_{p}} e_{i_{p}}^{(p)} t_{s}^{i_{p}}\right)
\end{aligned}
$$

Thus, since $t_{i}$ have no restrictions, $I$ has infinitely many strong jumping hyperplane.

We call Schwarzenberger bundle the vector bundle associated to $I$ (in fact in the case $p=2$ it is exactly the same introduced by Schwarzenberger in [10], see also [1]).

Proposition 2.10. Let $A$ be a boundary format matrix with $\operatorname{Det} A \neq 0$. If $A$ has $N \geq k_{0}+3$ strong jumping hyperplanes then it is an identity.

Proof. In the case $p=2$ the statement is proved in [1, Theorem 5.13] or in [14, Theorem 3.1]. Chosen $V_{0}$ and other two vector spaces among $V_{1}, \ldots, V_{p}$ (say $V_{1}$ and $V_{2}$ ), one may perform several elementary transformations with $V_{0}$ and all the others so that we get $A^{\prime} \in V^{\prime}{ }_{0} \otimes V_{1} \otimes V_{2}$ boundary format matrix with $\operatorname{Det} A^{\prime} \neq 0$ and $N^{\prime} \geq k_{0}^{\prime}+3$ strong jumping hyperplanes, then $A^{\prime}$ is an identity.

As in the above example, one can change the hyperplane giving the elementary transformation, so that for all $N$ strong jumping hyperplanes we get $t_{1}, \ldots, t_{N}$ distinct complex numbers and corresponding suitable basis of $V_{1}$ and $V_{2}$ :

$$
\begin{gathered}
\bar{e}_{0}^{(1)}, \ldots, \bar{e}_{k_{1}}^{(1)} \\
\bar{e}_{0}^{(2)}, \ldots, \bar{e}_{k_{2}}^{(2)}
\end{gathered}
$$


such that the hyperplanes are given by

$$
\sum_{i=0}^{k_{1}} \bar{e}_{i}^{(1)} t_{j}^{i} \quad \text { and } \quad \sum_{i=0}^{k_{2}} \bar{e}_{i}^{(2)} t_{j}^{i} \quad \text { for } j=1, \ldots N
$$

Now, changing $V_{1}$ and $V_{2}$ with the pairs $V_{1}, V_{j}(j=1, \ldots p)$ we get

$$
A:=\sum_{s=0}^{k_{0}} \bar{e}_{s}^{0} \otimes\left(\sum_{i_{1}=0}^{k_{1}} e_{i_{1}}^{(1)} t_{s}^{i_{1}}\right) \otimes \cdots \otimes\left(\sum_{i_{p}=0}^{k_{p}} e_{i_{p}}^{(p)} t_{s}^{i_{p}}\right)
$$

showing that $A$ is an identity.

Proposition 2.11. Two nondegenerate boundary format matrices having in common $k_{0}+2$ distinct strong jumping hyperplanes determine isomorphic Steiner bundles for every $j$.

Proof. In the case $p=2$ the statement is proved in [1, Theorem 5.3]. Chosen $V_{0}$ and other two vector spaces among $V_{1}, \ldots, V_{p}$ (say $V_{1}$ and $V_{2}$ ), one may perform several elementary transformations with $V_{0}$ and all the others so that we get $A^{\prime} \in$ $V^{\prime}{ }_{0} \otimes V_{1} \otimes V_{2}$ boundary format matrix with Det $A^{\prime} \neq 0$ and $N^{\prime}=k_{0}^{\prime}+2$ strong jumping hyperplanes, then $S_{A^{\prime}}^{(j)}$ is uniquely determined for every $j$. Now, changing $V_{1}$ and $V_{2}$ with the pairs $V_{1}$ and $V_{j}(j=2, \ldots, p)$ we detect all the 3 -dimensional submatrices of $A$ which give bundles uniquely determined, so also $S_{A}^{(j)}$ is uniquely determined for every $j$.

Remark 2.12. In the case $p=2$ we know that $k_{0}+2$ jumping hyperplanes give an existence condition for the bundles $S_{A}^{(j)}$ (they are logarithmic bundles, see [1]) but in the case $p \geq 3$ there is not an analog existence result.(The previous proposition gives only the uniqueness.)

The following is a classical result (see for instance [7, prop. 9.4, page 102], or [4, Theorem 6.8]).

Proposition 2.13. All nondegenerate matrices of type $2 \times k \times(k+1)$ are $\mathrm{GL}(2) \times$ $\mathrm{GL}(k) \times \mathrm{GL}(k+1)$ equivalent, or equivalently every surjective morphism of vector bundles on $\mathbb{P}^{1}$

$$
\mathcal{O}_{\mathbb{P}^{1}}^{k+1} \rightarrow \mathcal{O}_{\mathbb{P}^{1}}(1)^{k}
$$

is represented by an identity matrix.

We recall now the following

Proposition 2.14 ([1]). Let $A \in V_{0} \otimes \cdots \otimes V_{p} A$ be a $(p+1)$-dimensional matrix of boundary format the following conditions are equivalent:

(i) $A$ is an identity; 
(ii) there exist a vector space $U$ of dimension 2 and isomorphisms $V_{j} \simeq S^{k_{j}} U$ such that $A$ belongs to the unique one dimensional $\mathrm{SL}(U)$-invariant subspace of $S^{k_{0}} U \otimes \cdots \otimes S^{k_{p}} U$.

The equivalence between (i) and (ii) follows easily from the following remark: the matrix $A$ satisfies the condition (ii) if and only if it corresponds to the natural multiplication map $S^{k_{1}} U \otimes \cdots \otimes S^{k_{p}} U \rightarrow S^{k_{0}} U$ (after a suitable isomorphism $U \simeq U^{\vee}$ has been fixed). We notice that by the Clebsch-Gordan decomposition of the tensor product there is a unique $\mathrm{SL}(U)$-invariant map as above.

Remark 2.15. If $A$ is not an identity, an element $g \in \operatorname{Stab}(A)$ preserves a $(j)$-weak jumping hyperplane $h$ and it induces $\bar{g} \in \mathrm{SL}\left(V_{0} /\left\langle g\left(v_{0}\right)\right\rangle\right) \times \mathrm{SL}\left(V_{1}\right) \times \cdots \times \mathrm{SL}\left(V_{j} /\left\langle g\left(v_{j}\right)\right\rangle\right) \times$ $\cdots \times \mathrm{SL}\left(V_{p}\right)$ such that $g \cdot A$ projects to $\bar{g} \cdot A^{\prime}{ }_{j}$ and the elementary transformation behaves well with respect to the action of $g$.

For every integer $j$, let $D_{j \text {,strong }}(A)$ be the locus of $(j)$-strong directions of $A$ defined as

$$
\begin{aligned}
\left\{\left\langle v_{j}\right\rangle \in \mathbb{P}\left(V_{j}^{\vee}\right) \mid \forall i \neq j \exists v_{i} \in V_{i}\right. \text { such that } \\
\left.v_{0} \otimes \cdots \otimes v_{p} \text { is a strong jumping hyperplane for } A\right\} .
\end{aligned}
$$

We recall that (see for details [1]) for boundary format matrices the following conditions are equivalent

(i) $A \in V_{0} \otimes \cdots \otimes V_{p}$ is diagonal,

(ii) $\mathbb{C}^{*} \subset \operatorname{Stab}(A)$,

(iii) there exist a vector space $U$ of dimension 2, a subgroup $\mathbb{C}^{*} \subset \operatorname{SL}(U)$ and isomorphisms $V_{j} \simeq S^{k_{j}} U$ such that $A$ is a fixed point of the induced action of $\mathbb{C}^{*}$.

Then, the same proofs of Corollaries 6.9-6.10 and Lemmas 6.12-6.13 of [1] work also in the $(p+1)$-dimensional case, by replacing $V$ by $V_{j}$ and $W(S)$ by $D_{j \text {,strong }}(A)$. More precisely we have:

Corollary 2.16. Let $A$ be a boundary format nondegenerate matrix. If $\mathbb{C}^{*} \subset \operatorname{Stab}(A)$ then for every $j$ the $\mathbb{C}^{*}$-action on $V_{j}$ has exactly $k_{j}+1$ fixed points whose weights are proportional to $-k_{j},-k_{j}+2, \ldots, k_{j}-2, k_{j}$.

Remark 2.17. More in general, the $\mathbb{C}^{*}$-action on $V$ (where $V$ is a $n+1$-dimensional vector space) has exactly $n+1$ fixed points whose weights are proportional to $-n$, $-n+2, \ldots, n-2, n$ if and only if there exist a vector space $U$ of dimension 2 such that $\mathbb{C}^{*} \subset \mathrm{SL}(U)$ and $V \simeq S^{n} U$.

Corollary 2.18. Let $A$ be a boundary format nondegenerate matrix such that $\mathbb{C}^{*} \subset$ $\operatorname{Stab}(A)$. Then either $A$ is an identity or $D_{j, \text { strong }}(A)$ has only two closed points, namely the two fixed points of the dual $\mathbb{C}^{*}$-action on $\mathbb{P}\left(V_{j}^{\vee}\right)$ having minimum and maximum weights. 
Lemma 2.19. Let $U$ be a 2-dimensional vector space, and $\forall j C_{j} \simeq \mathbb{P}(U) \rightarrow \mathbb{P}\left(S^{k_{j}} U\right)$ be the $\mathrm{SL}(U)$-equivariant embedding (whose image is a rational normal curve). Let $\mathbb{C}^{*} \subset \mathrm{SL}(U)$ act on $\mathbb{P}\left(S^{k_{j}} U\right)$. We label the $k_{j}+1$ fixed points $P_{i}, i=-k_{j}+2 n$, $n=0, \ldots, k_{j}$, of the $\mathbb{C}^{*}$-action with an index proportional to its weight. Then $P_{-k_{j}}$, $P_{k_{j}}$ lie on $C_{j}$ and $P_{-k_{j}+2 n}=T^{n} P_{-k_{j}} \cap T^{k_{j}-n} P_{k_{j}}$, where $T^{n}$ denotes the $n$-dimensional osculating space to $C_{j}$.

Lemma 2.20. Let $A$ be a boundary format nondegenerate matrix. If there are two different one-parameter subgroups $\lambda_{1}, \lambda_{2}: \mathbb{C}^{*} \rightarrow \operatorname{Stab}(A)$ then $A$ is an identity.

Proof of Theorem 1.2. We proceed by induction on $k_{0}$.

If $k_{0}=2$ the theorem is true by Proposition 2.13 .

When $\operatorname{Stab}(A)^{0}$ contains only the identity the result is trivial hence we may suppose that $\operatorname{dim} \operatorname{Stab}(A)^{0} \geq 1$ then, according to [1, Theorem 2.4], the matrix $A$ is triangulable and there exists at least one strong jumping hyperplane $\mathcal{H}=v_{0} \otimes \cdots \otimes v_{p}$.

We may also suppose that the number of strong jumping hyperplanes is finite otherwise $\mathrm{A}$ is an identity (Proposition 2.10), hence $\mathcal{H}$ is $\operatorname{Stab}(A)^{0}$-invariant. Let $A_{1}^{\prime}$ be the image of $A$ by the elementary transformation associated to the (1)-weak jumping hyperplane defined by $\mathcal{H}$ (we choose $j=1$ to have simpler notations). The matrix $A_{1}^{\prime}$ belongs to $V_{0}^{\prime} \otimes V_{1}^{\prime} \otimes V_{2} \otimes \cdots \otimes V_{p}$ where $V_{0}^{\prime}=V_{0} /\left\langle v_{0}\right\rangle$ and $V_{1}^{\prime}=V_{1} /\left\langle v_{1}\right\rangle$, it is nondegenerate and of boundary format then, by induction, there exists a 2dimensional vector space $U$ such that

$$
V_{0}^{\prime} \simeq S^{k_{0}-1}(U), \quad V_{1}^{\prime} \simeq S^{k_{1}-1}(U) \quad \text { and } \quad V_{i}=S^{k_{i}}(U) \text { for all } i \geq 2
$$

and $\operatorname{Stab}\left(A_{1}^{\prime}\right)^{0} \subseteq \operatorname{SL}(U)$ (by using essentially the same argument we could work in $\left.\mathrm{GL}\left(V_{0}\right) \times \cdots \times \mathrm{GL}\left(V_{p}\right)\right)$.

Since $A_{1}^{\prime}$ is obtained from the matrix $A$ after the choice of two directions, any element which stabilizes $A$ also stabilizes $A_{1}^{\prime}$, so $\operatorname{Stab}(A)^{0} \subseteq \operatorname{Stab}\left(A_{1}^{\prime}\right)^{0}$. Hence $\operatorname{Stab}(A)^{0} \subseteq \operatorname{SL}(U)$ and $\operatorname{SL}(U)$ acts on $V_{i}$ according to $V_{i} \simeq S^{k_{i}} U$ for $i \geq 2$, by the inductive hypothesis.

Now, we claim that the action of $\operatorname{SL}(U)$ can be lifted to the whole $V_{0} \otimes \cdots \otimes V_{p}$.

Indeed, the above considered elementary transformation gives the decomposition $V_{0}=V_{0}^{\prime} \oplus \mathbb{C}$ and $V_{1}=V_{1}^{\prime} \oplus \mathbb{C}$.

If $\phi: \mathbb{C}^{*} \rightarrow \mathrm{GL}\left(V_{i}^{\prime}\right)$ is the natural action of $\mathbb{C}^{*} \subset \mathrm{SL}(U)$ on $V_{i}^{\prime}=S^{k_{i}-1} U$ (for $i=0,1)$ with $k_{i}$ fixed points having weights $-k_{i}+1,-k_{i}+3, \ldots, k_{i}-1$, we can construct an action $\psi: \mathbb{C}^{*} \rightarrow \mathrm{GL}\left(V_{i}^{\prime} \oplus \mathbb{C}\right)$ on $V_{i}$ defined by

$$
t \mapsto\left(\begin{array}{cc}
t^{-1} \phi(t) & 0 \\
0 & t^{k_{i}}
\end{array}\right)
$$

having $k_{i}+1$ fixed points with weights $-k_{i},-k_{i}+2, \ldots, k_{i}$. hence, by remark 2.17 , the statement follows.

In the case $\operatorname{Stab}(A)^{0}=\operatorname{SL}(2)$, the action of $\mathrm{SL}(U)$ satisfies definition 2.14, proving that $A$ is an identity. 
Now, as in [1], consider the Levi decomposition $\operatorname{Stab}(A)^{0}=M \cdot R$ where $\mathrm{R}$ is the radical and $\mathrm{M}$ is maximal semisimple. If $A$ is not an identity (i.e., $\left.\operatorname{Stab}^{0}(A) \neq \mathrm{SL}(2)\right)$ then $M=0$ and $\operatorname{Stab}(A)^{0}$ is solvable hence by the Lie Theorem it is contained (after a convenient basis has been chosen) in the subgroup of upper triangular matrices

$$
T=\left\{\left(\begin{array}{cc}
a & b \\
0 & \frac{1}{a}
\end{array}\right) \mid a \in \mathbb{C}^{*}, b \in \mathbb{C}\right\} .
$$

If there is a subgroup $\mathbb{C}^{*}$ properly contained in $\operatorname{Stab}(A)^{0}$ then there is a conjugate of $\mathbb{C}^{*}$ different from itself and this is a contradiction by the Lemma 2.20. If $\operatorname{Stab}(A)^{0}$ does not contain proper subgroups $\mathbb{C}^{*}$ then it is isomorphic to

$$
\mathbb{C} \simeq\left\{\left(\begin{array}{ll}
1 & b \\
0 & 1
\end{array}\right) \mid b \in \mathbb{C}\right\} .
$$

Remark 2.21. Throughout this paper we work only on nondegenerate matrices. Indeed, in the proofs we apply the induction strategy (hence the results of [1]) and the correspondence between matrices and vector bundles described in Proposition 1.4.

The characterization of the stabilizer of degenerate matrices is still an open problem. Another interesting problem is the study of the stabilizer of general multidimensional matrices (and not necessarily of boundary format).

\section{References}

[1] V. Ancona and G. Ottaviani, Unstable hyperplanes for Steiner bundles and multidimensional matrices, Adv. Geom. 1 (2001), 165-192.

[2] C. Dionisi, Multidimensional matrices and minimal resolutions of vector bundles, 2000.

[3] C. Dionisi and G. Ottaviani, The Binet-Cauchy theorem for the hyperdeterminant of boundary format multi-dimensional matrices, J. Algebra 259 (2003), 87-94.

[4] I. Dolgachev and M. Kapranov, Arrangements of hyperplanes and vector bundles on $\mathbf{P}^{n}$, Duke Math. J. 71 (1993), 633-664.

[5] I. M. Gel'fand, M. M. Kapranov, and A. V. Zelevinsky, Discriminants, resultants, and multidimensional determinants, Mathematics: Theory \& Applications, Birkhäuser Boston Inc., Boston, MA, 1994, ISBN 0-8176-3660-9.

[6] _ Hyperdeterminants, Adv. Math. 96 (1992), 226-263.

[7] J. Harris, Algebraic geometry, Graduate Texts in Mathematics, vol. 133, Springer-Verlag, New York, 1992, ISBN 0-387-97716-3.

[8] M. Maruyama, Elementary transformations in the theory of algebraic vector bundles, Algebraic Geometry (La Rábida, 1981), Lecture Notes in Math., vol. 961, Springer, Berlin, 1982, pp. 241266 .

[9] P. G. Parfenov, Orbits and their closures in the spaces $\mathbb{C}^{k_{1}} \otimes \cdots \otimes \mathbb{C}^{k_{r}}$, Mat. Sb. 192 (2001), 89-112. (English transl.)

[10] R. L. E. Schwarzenberger, Vector bundles on the projective plane, Proc. London Math. Soc. (3) 11 (1961), 623-640. 
[11] J.-P. Serre, Algèbre locale. Multiplicités, Cours au Collège de France, 1957-1958, rédigé par Pierre Gabriel. Seconde édition, 1965. Lecture Notes in Mathematics, vol. 11, Springer-Verlag, Berlin, 1965.

[12] J. Weyman and A. Zelevinsky, Singularities of hyperdeterminants, Ann. Inst. Fourier (Grenoble) 46 (1996), 591-644. (English, with English and French summaries)

[13] J. Vallès, Fibrés de Schwarzenberger et coniques de droites sauteuses, Bull. Soc. Math. France 128 (2000), 433-449.

[14] Nombre maximal d'hyperplans instables pour un fibré de Steiner, Math. Z. 233 (2000), $507-514$ 